\title{
Management of Facial Paralysis due to Extracranial Tumors
}

\author{
Michael Fritz, MD, FACS ${ }^{1}$ Bryan N. Rolfes, MD ${ }^{1}$ \\ 1 Section of Facial Plastic and Reconstructive Surgery, Cleveland Clinic \\ Head and Neck Institute, Cleveland, Ohio \\ Address for correspondence Michael Fritz, MD, FACS, Cleveland Clinic \\ Head and Neck Institute, 9500 Euclid Ave, Desk A71, Cleveland, Ohio \\ Facial Plast Surg 2015;31:110-116. \\ 44195 (e-mail: FRITZM1@ccf.org).
}

\section{Abstract \\ Keywords \\ - radical parotidectomy \\ - facial nerve grafting \\ - anterolateral thigh free flap}

Treatment of advanced parotid or cutaneous malignancies often requires sacrifice of the facial nerve as well as resection of the parotid gland and surrounding structures. In addition to considerations regarding reinnervation and dynamic reanimation, reconstruction in this setting must take into account unique factors such as soft tissue volume deficits and the high likelihood of adjunctive radiation therapy. Furthermore, considerations of patient comorbidities including advanced age and poor long-term prognosis often influence reconstructive modality. The optimal reconstructive technique would provide potential for restoration of facial tone and voluntary movement as well as immediate restoration of facial support and function. Beyond considerations of facial movement and rest position, restoration of lost soft tissue volume is critical to obtain facial symmetry. To control long-term volume in the setting of adjunctive radiation therapy, vascularized tissue is required. In this chapter, we describe a comprehensive approach to the management of radical parotidectomy and similar facial defects that addresses these concerns and also describes management strategies over time. Specific techniques employed include anterolateral thigh free flaps, nerve grafting utilizing motor nerves to the vastus lateralis muscle, and orthodromic temporalis tendon transfer. Further considerations relative to the eye, forehead, and long-term facial refinement are also discussed.
Facial nerve invasion mandates nerve sacrifice in up to $20 \%$ of malignant tumors involving the parotid gland; similarly, soft tissue and nerve resections are required with aggressive cutaneous malignancies of the head and neck. ${ }^{1}$ Beyond obvious effects of facial paralysis, tumor extirpation often results in profound soft tissue and even composite defects that can produce equally deforming and functional deficits. Optimal reconstruction replaces absent tissue with analogous structure to restore both symmetry and function while minimizing patient and donor site morbidity. Any technique applied in this setting must also take into account the high likelihood of adjunctive postoperative radiation therapy. Furthermore, in contrast to facial paralysis from nonmalignant etiologies, poor long-term prognosis and more advanced patient age and comorbidities mandate rapid restoration of form and function.

Options regarding rehabilitation of facial paralysis following nerve transection include grafting procedures, static suspension, regional muscle transfer, and/or free tissue transfer. While the former provides the best option for long-term recovery of tone and function, the effects of nerve grafting may not be noticeable for up to 12 months; this lag time becomes more significant in the setting of potential tumor recurrence or progression. Furthermore, with an average age of over 60 years, the patient with a parotid malignancy has less potential for a good outcome with nerve grafting alone. ${ }^{2,3}$ With these factors in mind, combined methods which both mitigate immediate functional deficits and allow for optimal long-term outcomes are ideal.

Methods to accomplish immediate rehabilitation at the time of facial nerve and parotid resection include both

Copyright $\odot 2015$ by Thieme Medical Publishers, Inc., 333 Seventh Avenue, New York, NY 10001, USA. Tel: +1(212) 584-4662.
DOI http://dx.doi.org/ 10.1055/s-0035-1549045. ISSN $0736-6825$. 
static suspension procedures and regional muscle transfer. By providing potential for both facial symmetry at rest and voluntary facial movement without creation of a secondary harvest site defect, orthodromic temporalis tendon transfer (OTTT) has been embraced as an effective technique in facial reanimation. ${ }^{4,5}$ In the setting of parotidectomy, easy access to the mandibular insertion of the temporalis tendon and the ability to perform tendon transfer without compromising nerve grafts/reinnervation are additional factors favoring this choice. While the degree of voluntary facial movement generated through this procedure is variable, surgical outcomes that establish immediate facial symmetry and support are nearly universal. ${ }^{6-8}$ These outcomes are in part due to the natural orientation of the temporalis tendon in a vector nearly identical to midface mimetic musculature. It is this ability to rapidly establish symmetry and maintain facial function with autogenous tissue (irrespective of radiation therapy) that has made OTTT the ideal method of reanimation in the oncologic setting.

Although free muscle transfer is embraced as the optimal reanimation technique as general approach to facial paralysis, this method is not typically considered as a component of primary rehabilitation in the oncologic setting. Delayed functional recovery and the unpredictability of outcomes regarding innervation and overall function of irradiated flaps outweigh any possibility of improved dynamic results. This option may be considered in delayed fashion in a favorable subset of patients (e.g., young with good oncologic prognosis).

Regardless of facial nerve involvement, free vascularized tissue transfer allows for accurate and stable soft tissue volume correction resistant to the effects of radiation therapy; it has thus become the method of choice for facial defect reconstruction following parotidectomy. More traditional techniques of contour correction involving locoregional muscle transfer (e.g., platysma or sternocleidomastoid muscle flaps), free autologous tissue, and allogeneic grafts can be compromised by long-term atrophy and spontaneous or radiation-induced resorption. ${ }^{9-11}$ Furthermore, evidence of neovascularization of surrounding tissues after transfer of a vascular pedicle has been well established. ${ }^{12}$ These data lend credence to a potential radioprotective effect on surrounding structures (e.g., mandible, adjacent muscles/nerves) when free tissue transfer is employed.

Given its very low donor site morbidity and ability to provide ample vascularized tissue that can be easily contoured for defect correction, the anterolateral thigh (ALT) free flap is optimally suited for parotidectomy defect reconstruction. Furthermore, ALT flaps allow for easy twoteam surgery and provide additional grafting material (nerve and fascia lata) via a single harvest site. ${ }^{7}$ While other flap harvest sites have been described (e.g., radial forearm), these are less ideal due to limited tissue for contouring, greater harvest site morbidity, and/or creation of more visible scars. Importantly, free muscle flaps are not utilized, as continued atrophy over time precludes precise long-term volume control.

\section{Surgical Management}

\section{Preoperative Considerations and Planning}

Patients are ideally referred preoperatively for reconstructive considerations regarding anticipated surgery for advanced cutaneous cancers or suspected parotid malignancy. If oncologic factors merit consideration of facial nerve sacrifice, all aspects of facial reconstruction including vascularized tissue contouring, facial nerve grafting, and dynamic facial reanimation are discussed. Perioperative expectations and anticipated realistic long-term outcomes are reviewed in detail.

Patients who present with paralysis involving the upper division that suffer lagophthalmos are sized for an appropriate upper eyelid weight; upper lid weight placement and ectropion repair (lateral tarsal strip) are typically performed under local anesthesia prior to tumor resection to minimize the duration under general anesthesia. Similarly, periorbital reanimation in those with intact function who undergo facial nerve sacrifice intraoperatively is typically not performed at the time of tumor resection but rather within 2 to 3 weeks postoperatively under local anesthesia. This method both reduces anesthesia time and allows for more accurate weight sizing and ectropion repair.

\section{Acute Defect Management}

\section{Graft Harvest}

Our method of immediate management of radical parotidectomy defects at the time of oncologic resection addresses the three goals of reinnervation, reanimation, and facial contour restoration. Unless otherwise precluded, the left thigh is always chosen as the donor site to return patients to full function (including driving a car) as soon as possible. This donor site provides three key elements: (1) anterolateral free flap, (2) vastus lateralis motor nerve grafts, and (3) fascia lata for lower lip suspension (performed in conjunction with OTTT). Again, procedures related to lagophthalmos, ectropion repair, and brow ptosis are typically performed under local anesthesia or sedation before or following tumor resection.

While oncologic resection is underway, Doppler probe is used to locate and mark perforators near the midpoint between superolateral patella and anterior superior iliac spine, and a skin flap with appropriate size and bulk is harvested using described harvest technique. ${ }^{13}$ While primary closure is ensured with flap design, skin is always included in the flap regardless of whether a cutaneous defect is anticipated. This allows for more facile and less traumatic flap manipulation, and provides a vascularized dermal graft that amplifies available tissue volume in thin patients. As the deeper soft tissues are divided, an area of fascia lata greater in dimension than the skin paddle is included to capture additional vascularized tissue which can be employed for precise contour correction. Critically, flap harvest is performed with complete sparing of the vastus lateralis muscle (perforator flap technique) to maximize pedicle length and to avoid importation of denervated muscle into the defect. This muscle will atrophy over time and thus compromise long-term control of tissue volume. 


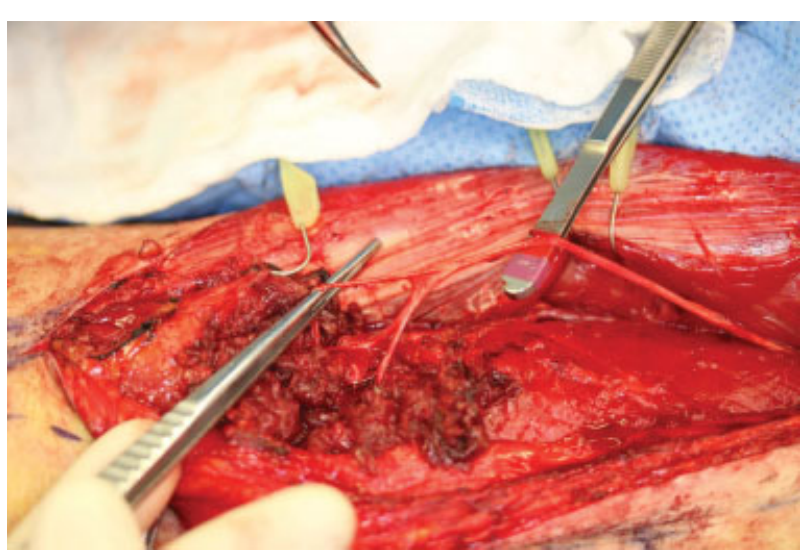

Fig. 1 Typical length and branching pattern of motor nerve to vastus lateralis (MNVL) following ALT perforator flap harvest.

At the time of pedicle dissection, motor nerve to the vastus lateralis (MNVL) muscle will be encountered. These nerves are extremely redundant with multiple branches and potential length is equivalent to sural nerve, lateral femoral cutaneous nerve, or medial antebrachial cutaneous nerve grafts (-Fig. 1). ${ }^{14}$ Furthermore, because of redundancy proximally, sacrifice of branches of the MNVL is routinely performed during ALT pedicle harvest (irrespective of grafting needs) without impairing postoperative function. ${ }^{15}$ Animal evidence suggests that an additional advantage of improved rapidity and quality of neural regeneration may be achieved through use of a nerve graft with matching motor (rather than sensory) modality. ${ }^{16,17}$

Once tumor margin clearance and adjunctive ablative procedures are completed, the ALT vascular pedicle is divided, the MNVL graft is harvested, and a 2-cm wide and 10-cm long slip of fascia lata is also removed from the harvest site laterally. All elements are then brought to the head and neck region for reconstruction. The ALT is typically revascularized first and then temporarily draped inferiorly away from the parotid bed while nerve grafting and OTTT are underway (-Fig. 2). This allows for a period of open monitoring of the revascularized flap to confirm vascular integrity.

\section{Reanimation}

Attention is focused next on immediate dynamic reanimation of the face with OTTT. In contrast to independent OTTT procedures, the mandible and temporalis tendon are readily accessible from a lateral approach through the ablative access incisions. If masseter muscle has not been resected, this is elevated off the lateral mandible and retracted superiorly to expose the coronoid process. Care is taken to avoid injury to the motor nerve to the masseter muscle, and in some instances, this nerve is isolated for later use. Thin insertions of the temporalis tendon on the lateral coronoid are elevated and the tendon on the medial aspect is carefully preserved via subperiosteal elevation and placement of a right angle hemostat around the deep surface of the bone. The coronoid is then divided with a sagittal saw, exposing the underlying
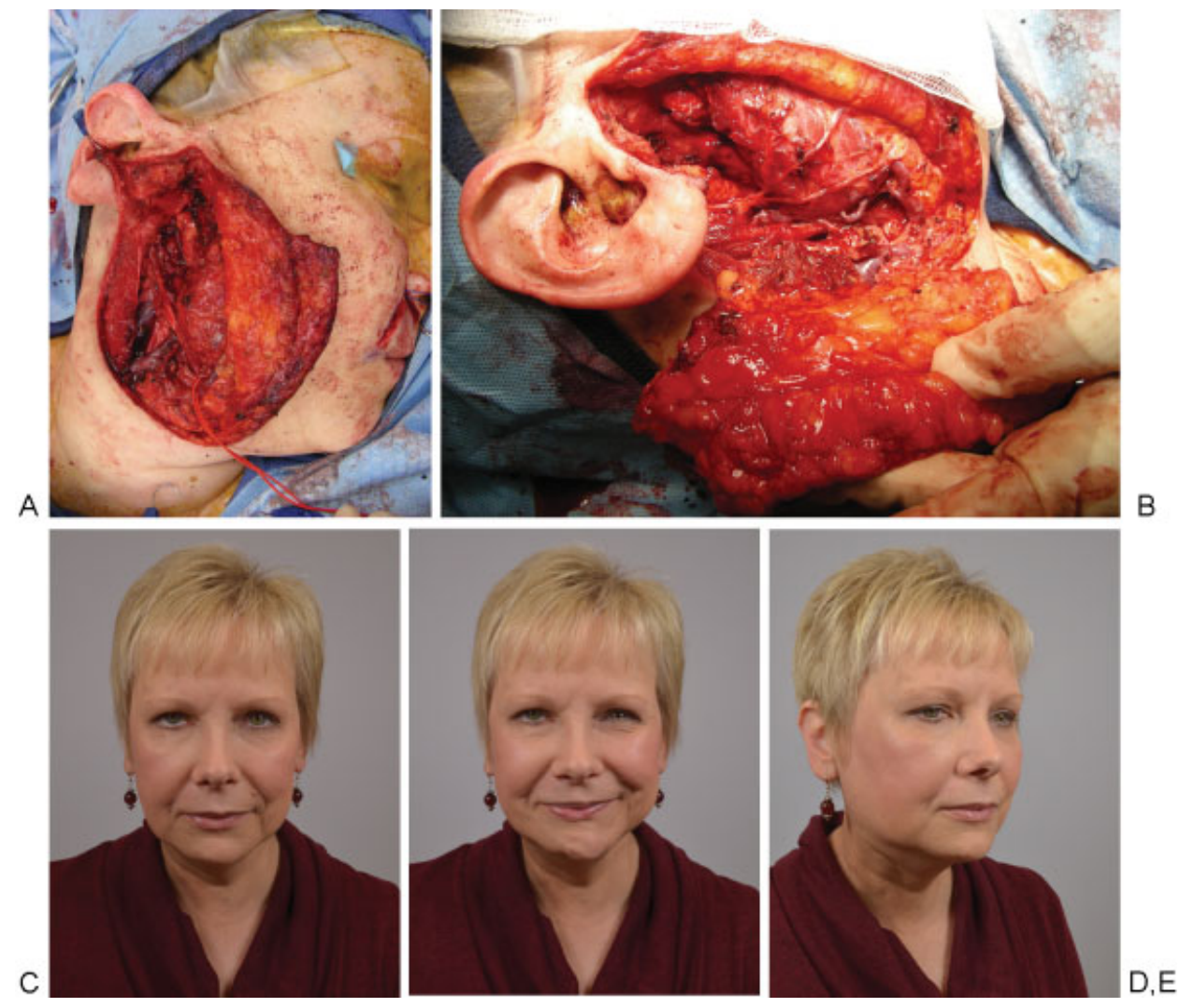

Fig. 2 The defect from radical parotidectomy (A), the defect with nerve grafts in place and revascularized anterolateral thigh (B), and the postsurgical result at 1 year (C-E). 
temporalis tendon. It is the portion of the tendon attached on the medial coronoid base and also to the mandibular angle region that provides optimal length and obviates the need for fascia lata extension (except for lower lip suspension). It is the authors' opinion that minimizing use of extension grafts is a key to maintaining reliable movement and position following postoperative radiation therapy.

Four to five sutures (4.0 PDS) are then placed in horizontal mattress fashion in the most robust portions of the divided temporalis tendon with the greatest length. Remaining tendon attached to the proximal coronoid is elevated off of the bone, which is then discarded. Importantly, this proximal tendon is not employed in the transfer, as reach is limited. At this point, it is also important to confirm that no remaining temporalis is attached to the mandible (i.e., the tendon moves freely).

A nasolabial fold incision is then created to allow tendon passage. In patients with a defined fold, the incision is created from $1 \mathrm{~cm}$ inferior to the alar base to $1 \mathrm{~cm}$ above the commissure. Blunt and sharp dissection is then performed through soft tissue and muscle, sparing neurovascular pedicles, creating a tunnel to allow for tendon passage, yet not interrupting the potential for reinnervation effect. The sutures are then passed medial to the masseter muscle, maintaining their orientation, and secured directly to the orbicularis/superficial muscular aponeurotic system (SMAS) extending along the length of the nasolabial fold ( - Fig. $\mathbf{3}$ ). The inferior-most or shortest suture/tendon slip is attached to the fascia lata lip suspension.

The lower lip suspension was added to OTTT technique to improve lip competence and mitigate volume loss due to orbicularis atrophy over time. A midline lower lip incision is performed and carried into the mid portion of the orbicularis marginalis muscle. A slip of fascia lata typically 1 to $1.5 \mathrm{~cm}$ wide is then trimmed and passed through a tunnel created within the central orbicularis from the midline to the inferior aspect of the nasolabial fold incision (-Fig. 4). Fascia lata is then secured medially to the midline orbicularis muscle and laterally to the temporalis tendon; tension is adjusted, so the lip is well supported without external distortion. This added lip suspension technique allows the dynamic tendon to work with the functional contralateral orbicularis and has significantly improved long-term function and appearance; it has obviated lower lip wedge resections previously performed (after longterm follow-up) due to lip lengthening and atrophy (-Fig. 5).

If oncologic management necessitates resection of the temporalis muscle, static facial suspension is performed with fascia lata. When flap and pedicle geometry permit, vascularized fascia lata is employed. However, nonvascularized suspension is performed if necessary to accomplish both precise midface position control and contour correction. Again given considerations of imminent adjunctive treatment, autogenous tissue suspension is preferred.

\section{Nerve Grafting}

After OTTT is completed, attention is focused on nerve grafting. The graft harvested from the thigh is tailored to length and number of branches required to span from proximal facial nerve to preserved distal branches. Ideally, all available

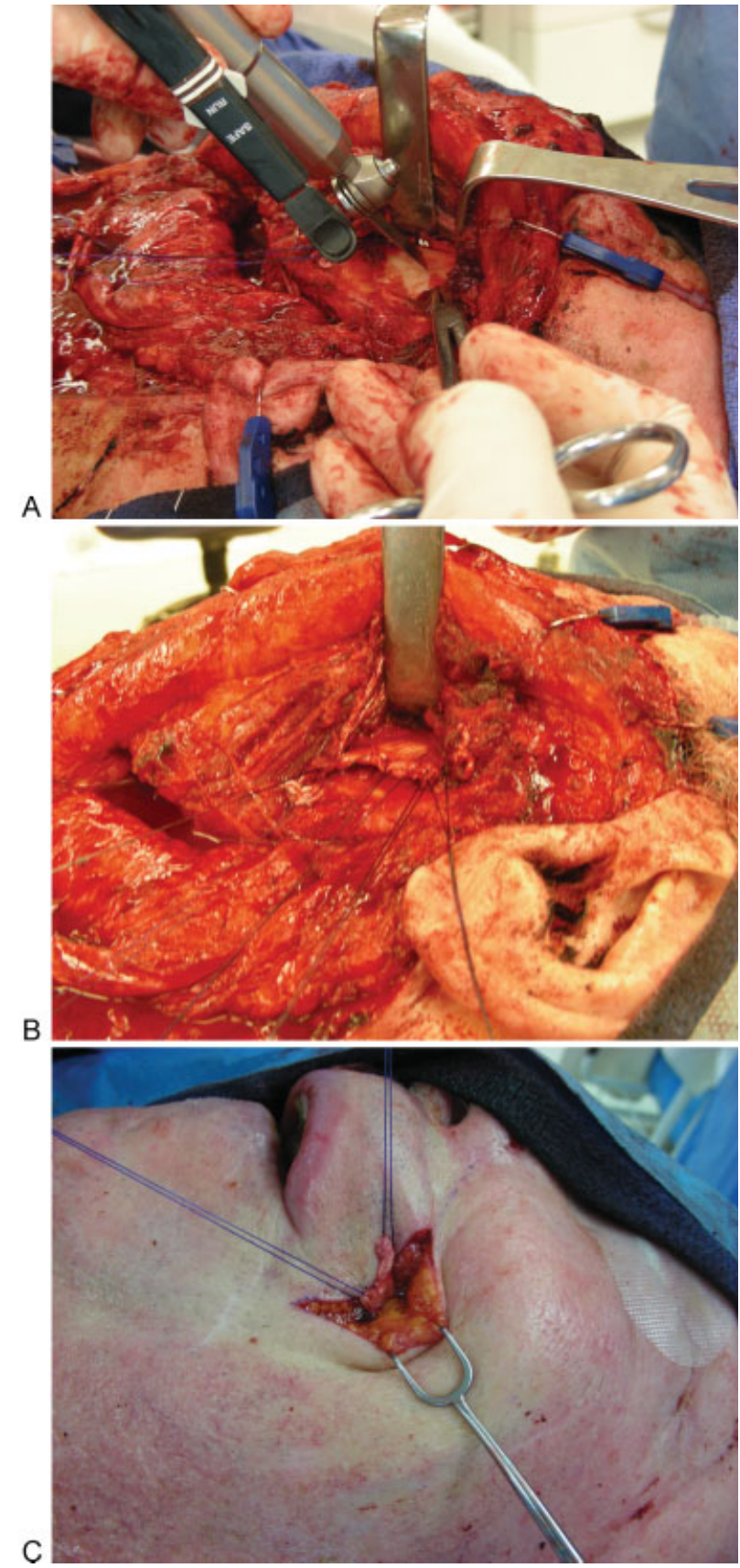

Fig. 3 Lateral exposure of coronoid (A). Horizontal mattress sutures placed in temporalis tendon (B). Tendon without extension reaching to oral commissure (C).

branches are grafted to optimize potential for facial tone and volume maintenance; the degree of anticipated nerve recovery in this population (typically House-Brackmann 3-4) mitigates concerns of synkinesis. ${ }^{18}$ If the latter develops over time, this is typically well controlled with Botox injection. In younger patients (e.g., younger than 40 years) in which more robust recovery is anticipated, branches are grafted and considerations of either free dynamic muscle transfer or OTTT are deferred until extent of recovery is defined by full reinnervation.

If the proximal facial nerve is unavailable for grafting and yet distal branches are well defined and free of tumor, the 

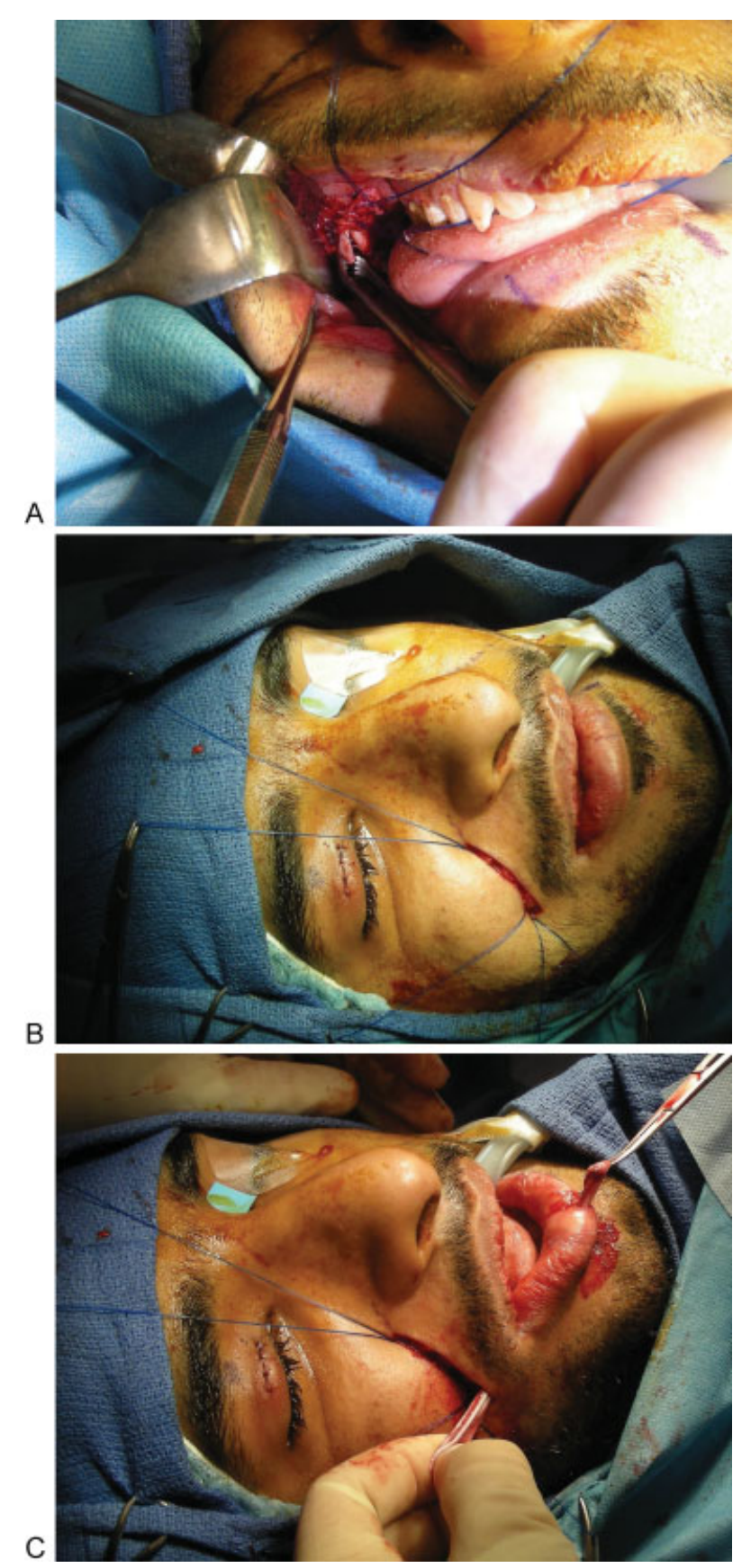

Fig. 4 Transoral exposure of coronoid (A). Sutures drawn through nasolabial incision (B). Fascia lata lower lip extension (C).

motor nerve to the masseter muscle can be employed for motor input. The nerve is isolated using the subzygomatic triangle technique described by Boahene and MNVL grafts are coapted to span from the masseteric nerve to distal facial branches. ${ }^{19}$ This reinnervation method is considered in patients who have been paralyzed for less than 12 months prior to tumor resection.

\section{Contour Restoration and Skin Replacement}

The previously vascularized ALT free flap is now draped over the nerve anastomosis within the parotid bed and the flap is circumferentially suspended to SMAS and deep soft tissues. If
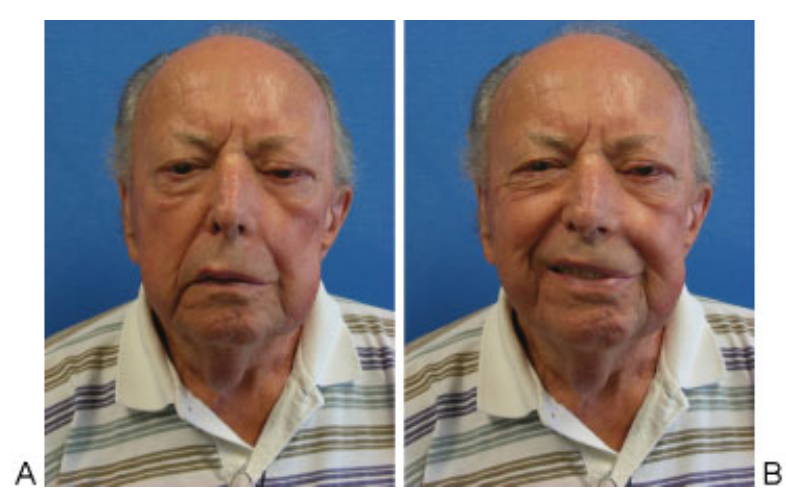

Fig. 5 (A, B) Demonstration of dynamic result after orthodromic temporalis tendon transfer.

a neck dissection has been performed, the flap is oriented for appropriate volume correction in this region as well. Skin and subcutaneous tissue are removed as required to establish symmetric contour with a slight overcorrection due to flap edema from harvest. Gross overcorrection is not performed, as the vascularized soft tissue volume remains stable over time. ${ }^{20}$ If flap orientation and soft tissue requirements allow, a small cutaneous monitoring segment is retained and ideally secured to native skin flaps posterior to the lobule.

Obviously, external skin defect reconstruction with color mismatched ALT skin is not optimal. However, full restoration of large areas of skin with cervicofacial flaps often requires extensive dissection and skin tension than can obscure precise volume correction and facial suspension. Vascularity of regional skin flaps is further compromised by nasolabial access incisions (if OTTT performed), and aggressive suspension may result in wound-healing issues. Considering these issues, cutaneous defects are addressed by cervicofacial advancement alone when amenable or more commonly a free flap skin paddle reduced in size as much as possible by locoregional advancement. If an aesthetic issue, mismatched skin is removed following full completion of treatment with additional advancement techniques or more commonly serial excision of the free flap. The latter is often performed under local anesthesia in the office setting.

\section{Postoperative Management}

Patients typically are managed in the hospital setting until neck drains are removed. If no neck dissection has been performed, they are often discharged home within 2 to 3 days with aggressive eye care. Drains in the ALT harvest site are removed with sutures around 1 week after surgery. For those patients who did not present with preoperative paralysis, eyelid weight sizing is performed and planning is begun for lid loading and ectropion repair at $\sim 2$ to 3 weeks after oncologic resection. Given the advantages of less visibility and extrusion risk, platinum chains are placed when long-term paralysis is anticipated. ${ }^{21,22}$ In contrast, due to greater ease of removal and lower expense, gold weights are still employed for eye protection when functional recovery is anticipated. 
If brow ptosis is symptomatic to the point of visual field compromise, browlift is performed at the same setting as periorbital reanimation. More commonly, this procedure is deferred until full completion of adjunctive therapy. In this patient population with typical preexisting forehead rhytids, the mid-forehead browlift technique, performed as described by Quatela et al, is an ideal method for facile and lasting brow correction. ${ }^{23}$ The scar, if visible, mimics contralateral rhytids and is superior to that created by other external approaches. Importantly, browlift is performed in a conservative fashion to avoid compromise of eye closure.

\section{Posttreatment and Long-Term Management}

For those individuals managed at the time of oncologic resection, refinement of lower eyelid position, brow correction, and contour adjustment may be required. The latter is deferred until resolution of treatment-related edema-typically 6 to 12 months after completion of adjunctive therapy. Further efforts to optimize are usually deferred until full reinnervation has occurred (18-24 months postoperative) and include Botox injection and rarely digastric muscle transposition. ${ }^{24}$

\section{Secondary Reconstruction}

For patients referred after treatment, initial priority is obviously given to eye protection and expedited management is performed prior to any planned postoperative radiation therapy. Additional procedures are deferred until 2 to 3 months after completion of therapy. At this point, browlift, any needed eyelid refinement, and OTTT are planned at the same operative setting.

Secondary OTTT is performed via a combined transoral and transfacial approach. Addition of the transoral exposure allows for capture of greater lengths of temporalis tendon which obviates the need for fascia lata extension grafts (except for lower lip suspension). It is the authors' opinion that limiting nonvascularized tissue grafts and creating direct tendon apposition to perioral structures help avoid the higher infection rate and decreased excursion seen in this patient population. ${ }^{25}$ In this approach, a 2 - to 3 -cm mucosal access incision is performed over the coronoid process and a supraperiosteal dissection is performed, exposing the longer slips of temporalis tendon on the medial coronoid and mandibular angle (-Fig. 4). Sutures are placed and coronoidectomy is performed utilizing a reciprocating saw. Tendon slips are passed through the nasolabial fold incision, and nasolabial and lower lip suspension is performed in identical fashion to the lateral approach as described previously. Notably, care is taken to limit subperiosteal dissection over the irradiated mandible, and aggressive intraoperative and postoperative antibiotic regimens are followed to avoid complications of infection or osteoradionecrosis.

Secondary contour deformities present a more difficult secondary challenge in an irradiated and scarred operative
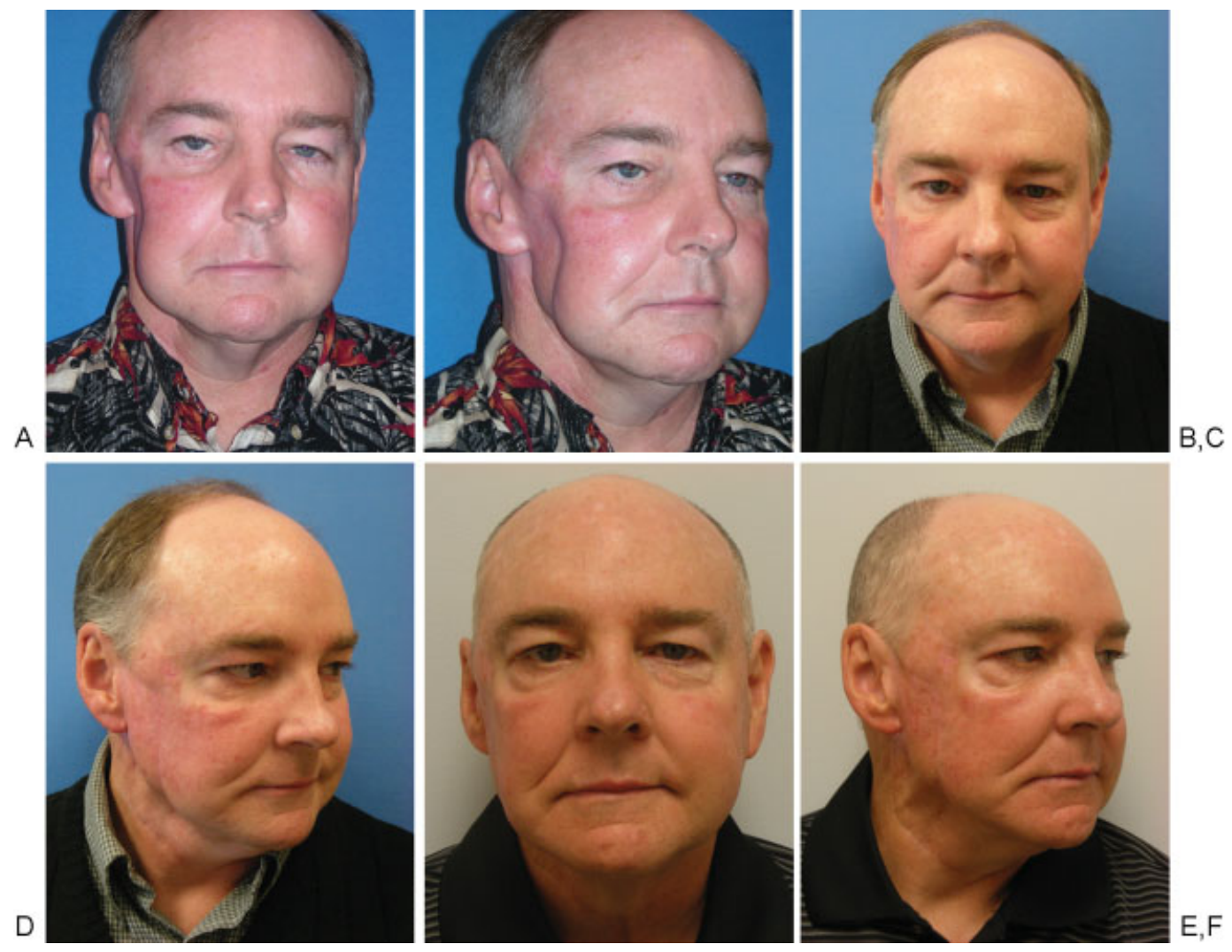

Fig. 6 (A, B) Radical parotidectomy defect 2 years posttreatment after orthodromic temporalis tendon transfer only (declined primary contour correction). (C, D) Result at 1 year after contour correction with anterolateral thigh flap. (E, F) Result at 5 years. 
bed, and correction is typically deferred for at least 12 months to allow optimal tissue recovery. These defects are amenable to secondary vascularized soft tissue reconstruction in which a subcutaneous pocket is created and the graft is oriented with percutaneous bolster sutures. Flap vascularization is typically performed using minimal access approaches for vessel exposure, thus minimizing patient morbidity and length of hospital admission (-Fig. 6). ${ }^{26}$

\section{Conclusion}

Optimal oncologic management of advanced parotid and cutaneous malignancies may necessitate aggressive tissue resection including facial nerve sacrifice. Management of this unique subset of patients requires consideration of age, comorbidities, planned adjunctive therapy, and overall prognosis. Furthermore, deformities caused by unaddressed volume deficits can rival those created by facial paralysis itself. With these factors in mind, optimal management of these patients requires a tripartite approach combining volume correction with vascularized ALT flaps, immediate dynamic reanimation with OTTT, and reinnervation with nerve grafts when possible. By following these principles, acceptable function, aesthetic outcomes, and quality of life can be preserved.

\section{References}

1 Theriault C, Fitzpatrick PJ. Malignant parotid tumors. Prognostic factors and optimum treatment. Am J Clin Oncol 1986;9(6):510-516

2 Brown PD, Eshleman JS, Foote RL, Strome SE. An analysis of facial nerve function in irradiated and unirradiated facial nerve grafts. Int J Radiat Oncol Biol Phys 2000;48(3):737-743

3 Reddy PG, Arden RL, Mathog RH. Facial nerve rehabilitation after radical parotidectomy. Laryngoscope 1999;109(6):894-899

4 Cannady SB, Seth R, Fritz MA, Alam DS, Wax MK. Total parotidectomy defect reconstruction using the buried free flap. Otolaryngol Head Neck Surg 2010;143(5):637-643

5 Boahene KD. Dynamic muscle transfer in facial reanimation. Facial Plast Surg 2008;24(2):204-210

6 Byrne PJ, Kim M, Boahene K, Millar J, Moe K. Temporalis tendon transfer as part of a comprehensive approach to facial reanimation. Arch Facial Plast Surg 2007;9(4):234-241

7 Revenaugh PC, Knott PD, Scharpf J, Fritz MA. Simultaneous anterolateral thigh flap and temporalis tendon transfer to optimize facial form and function after radical parotidectomy. Arch Facial Plast Surg 2012;14(2):104-109

8 Sidle DM, Fishman AJ. Modification of the orthodromic temporalis tendon transfer technique for reanimation of the paralyzed face. Otolaryngol Head Neck Surg 2011;145(1):18-23
9 Gooden EA, Gullane PJ, Irish J, Katz M, Carroll C. Role of the sternocleidomastoid muscle flap preventing Frey's syndrome and maintaining facial contour following superficial parotidectomy. J Otolaryngol 2001;30(2):98-101

10 Chandarana S, Fung K, Franklin JH, Kotylak T, Matic DB, Yoo J. Effect of autologous platelet adhesives on dermal fat graft resorption following reconstruction of a superficial parotidectomy defect: a double-blinded prospective trial. Head Neck 2009;31(4):521-530

11 Davis RE, Guida RA, Cook TA. Autologous free dermal fat graft Reconstruction of facial contour defects. Arch Otolaryngol Head Neck Surg 1995;121(1):95-100

12 Pribaz JJ, Fine NA. Prefabricated and prelaminated flaps for head and neck reconstruction. Clin Plast Surg 2001;28(2):261-272, vii

13 Wong CH, Wei FC. Anterolateral thigh flap. Head Neck 2010;32(4): 529-540

14 Revenaugh PC, Knott PD, McBride JM, Fritz MA. Motor nerve to the vastus lateralis. Arch Facial Plast Surg 2012;14(5):365-368

15 Casey WJ III, Rebecca AM, Smith AA, Craft RO, Hayden RE, Buchel EW. Vastus lateralis motor nerve can adversely affect anterolateral thigh flap harvest. Plast Reconstr Surg 2007;120(1):196-201

$16 \mathrm{Chu} \mathrm{TH}, \mathrm{Du} \mathrm{Y}, \mathrm{Wu}$ W. Motor nerve graft is better than sensory nerve graft for survival and regeneration of motoneurons after spinal root avulsion in adult rats. Exp Neurol 2008;212(2): 562-565

17 Moradzadeh A, Borschel GH, Luciano JP, et al. The impact of motor and sensory nerve architecture on nerve regeneration. Exp Neurol 2008;212(2):370-376

18 Ozmen OA, Falcioni M, Lauda L, Sanna M. Outcomes of facial nerve grafting in 155 cases: predictive value of history and preoperative function. Otol Neurotol 2011;32(8):1341-1346

19 Collar RM, Byrne PJ, Boahene KD. The subzygomatic triangle: rapid, minimally invasive identification of the masseteric nerve for facial reanimation. Plast Reconstr Surg 2013;132(1):183-188

20 Higgins KM, Erovic BM, Ravi A, et al. Volumetric changes of the anterolateral thigh free flap following adjuvant radiotherapy in total parotidectomy reconstruction. Laryngoscope 2012;122(4): 767-772

21 Silver AL, Lindsay RW, Cheney ML, Hadlock TA. Thin-profile platinum eyelid weighting: a superior option in the paralyzed eye. Plast Reconstr Surg 2009;123(6):1697-1703

22 Bianchi B, Ferri A, Leporati M, et al. Upper eyelid platinum chain placement for treating paralytic lagophthalmos. J Craniomaxillofac Surg 2014;42(8):2045-2048

23 Cook TA, Brownrigg PJ, Wang TD, Quatela VC. The versatile midforehead browlift. Arch Otolaryngol Head Neck Surg 1989; 115(2):163-168

24 Conley J, Baker DC, Selfe RW. Paralysis of the mandibular branch of the facial nerve. Plast Reconstr Surg 1982;70(5):569-577

25 Griffin GR, Abuzeid W, Vainshtein J, Kim JC. Outcomes following temporalis tendon transfer in irradiated patients. Arch Facial Plast Surg 2012;14(6):395-402

26 Cannady SB, Rosenthal EL, Knott PD, Fritz M, Wax MK. Free tissue transfer for head and neck reconstruction: a contemporary review. JAMA Facial Plast Surg 2014;16(5):367-373 